\title{
Advantages of axially aligned crystals used in positron production at future linear colliders
}

\author{
X. Artru, ${ }^{1}$ R. Chehab,${ }^{2}$ M. Chevallier, ${ }^{1}$ and V. Strakhovenko ${ }^{3, *}$ \\ ${ }^{1}$ IPN-Lyon, IN2P3/CNRS et University Claude Bernard, 69622 Villeurbanne, France \\ ${ }^{2}$ LAL, IN2P3/CNRS et University de Paris-Sud, BP 34-91898, Orsay cedex, France \\ ${ }^{3}$ Budker-INP, 11 Ac. Lavrentyeva, 630090, Novosibirsk, Russia \\ (Received 1 July 2003; published 22 September 2003)
}

\begin{abstract}
The characteristics of the electron-photon showers initiated by 2 to $10 \mathrm{GeV}$ electrons aligned along the $\langle 111\rangle$ axis of tungsten crystals are compared with those for the amorphous tungsten. In this energy range, as known, the positron yield at the optimal target thicknesses is larger in a crystal case only by several percent. However, the amount of the energy deposition in a crystal turns out to be considerably (by 20\%-50\%) lower than in an amorphous target providing the same positron yield, while the peak energy-deposition density is approximately of the same magnitude in both cases.
\end{abstract}

DOI: 10.1103/PhysRevSTAB.6.091003

PACS numbers: 07.77.Ka, 12.20.Ds, 03.65.Sq

\section{INTRODUCTION}

High energy $e^{+} e^{-}$colliders planned to explore physics at a $\mathrm{TeV}$ scale will operate at very high luminosities. Therefore, a very intense electron beam should be used to produce positrons in a target. A substantial part of the primary beam power (for example, more than $30 \%$ in the JLC project) will be dissipated in the target, causing serious thermal and radioactivity problems, as already observed in the SLC target.

The thermal problem, for its part, has two physical aspects: (i) average total deposited power, (ii) instantaneous energy-deposition density (EDD). The average deposited power can be handled, e.g., by rotating the target and removing the excess heat through water cooling as used at SLC. The deposited energy comes in pulses and is very nonuniformly distributed over the target volume. More precisely, the EDD is maximal on the beam axis and increases with the depth. At each pulse, a sudden mechanical stress appears due to the thermal gradient, which may break the target. Basing on the analysis of the SLC damaged target (see [1]), it is now adopted that the peak energy-deposition density (PEDD) should not exceed $35 \mathrm{~J} / \mathrm{g}$ to ensure a sufficiently long term of safe operation. One can reduce the PEDD by increasing the beam size, but this solution is limited by the geometrical acceptance of the $e^{+}$collecting system following the target. A solution for both problems adopted in JLC and NLC designs is to use several targets in parallel. But, in any case, the total deposited power and the PEDD should both be lowered as much as possible at a given positron yield.

As known, crystal targets may provide some advantages over conventional amorphous ones. In the present paper, the total deposited energy and the distribution of its density over the target volume are calculated for

\footnotetext{
*Corresponding author.

Email address: v.m.strakhovenko@inp.nsk.su
}

crystal and amorphous tungsten targets using the parameters of CLIC [2], NLC [3], and JLC [4]. Thereby, a possible gain for these projects resulting from the use of crystal targets in the positron source is estimated.

The formation of electromagnetic showers in aligned single crystals was actively studied during the last decade. The first experimental investigation of such showers has been performed in [5] at a very high energy of incident electrons. Corresponding theoretical studies were started with [6] where an analytic solution of the problem was obtained, assuming that energies of all charged particles and photons involved are very high. This limitation was surmounted in [7] by suggesting a specific form of the radiation spectrum at axial alignment and performing corresponding simulations. Using this approach, the results of [5] for the Ge crystal had been reproduced in [8]. The results of [7] are consistent with those of [9] where another approach was used to obtain the radiation spectrum. In $[7,9,10]$, the shower characteristics, such as spectral-angular distributions of photons and positrons as well as the amount of energy deposition, have been obtained depending on the kind and thickness of the crystal targets.

Investigations of the specific shower formation give good grounds for the idea proposed in [11], to substitute in a positron source an axially aligned crystal target for an amorphous one, as the enhancement of the radiation intensity is maximal just at the axial alignment. In further experiments (see [12-17]) using 1.2-10 GeV electrons aligned to the $\langle 111\rangle$ axis of tungsten crystals, measurements of some shower characteristics were already aimed to the development of a crystal-assisted positron source. Theoretical estimations performed in [18] display a rather good agreement with results of recent experiments [14-17]. So, we can rely on our understanding of the physics of shower formation and on numerical results, at least for tungsten crystals in the energy range of incident electrons below $10 \mathrm{GeV}$. Note that just this energy range is proposed in future linear collider projects $(2,6.2$, 
and $10 \mathrm{GeV}$ correspondingly for CLIC, NLC, and JLC) and is considered here.

According to $[7,9,10]$, the maximal positron yield from a crystal target is always higher than that from an amorphous one and the excess goes up when the electron energy increases. However, the magnitude of such an enhancement is small, less than $14 \%$ even at $10 \mathrm{GeV}$. As shown in the present paper, the most pronounced advantage of crystal targets appears in a considerable (by a factor of 2 at $10 \mathrm{GeV}$ ) decrease of the energy deposition. Qualitative arguments which explain this fact are given below. As for the peak energy-deposition density, it is approximately of the same magnitude in the crystal and in the amorphous cases. For accurate studies of thermal effects, some improvements have been performed in the computer code developed in $[7,10]$. The updated version of the code is used to study both crystal and amorphous cases.

\section{ENERGY DEPOSITION IN CRYSTAL AND AMORPHOUS TARGETS}

In the energy range under consideration we are dealing with a "soft" (see [6]) shower when pair production is entirely due to the conventional Bethe-Heitler mechanism, while the crystal structure reveals in a considerable enhancement of the radiation intensity and a softness of the photon spectra. Remember that this enhancement decreases when the particle energy does so as the shower develops. Starting with some depth $L_{0}$ (see discussion in $[7,10])$, further development of the shower proceeds more or less in the same way for any (crystal or amorphous) type of the remaining part of the target. For the sake of simplicity, calculations are performed here for the allcrystal targets. However, they may serve as a good estimate for hybrid targets of the same total thickness and with a crystal-part length of the order of $L_{0}$. Let us remind one that a hybrid target consists of a photon radiator made of a crystal followed by a pair converter made of an amorphous piece.

From Fig. 1 , a value of $L_{0} \gtrsim 0.2 \mathrm{~cm}\left(0.57 X_{0}\right)$ can be chosen for $10-\mathrm{GeV}$ electrons, since the fraction of the total energy carried by photons $(\sim 0.72)$ has already been saturated at this depth and the mean energy of the primary electron is sufficiently low to eliminate crystal effects in the last part. Such a saturation takes place in amorphous targets as well, but with a lower conversion level ( $\sim 0.59)$ and at substantially larger depth $\left(\sim 5 L_{0}\right.$ at $10 \mathrm{GeV}$ ). Only a small part (less than $0.4 \%$ in the above example) of the beam power is deposited over $L_{0}$ and the energy-deposition density is much less (about 8 times at $10 \mathrm{GeV}$ ) than its peak value. So, the crystal part of a hybrid target is not exposed to serious thermal effects which appear at larger depths in the later stage of the shower development.

From calculations performed in $[7,10]$, the energy deposition in equivalent (providing the same positron yield)
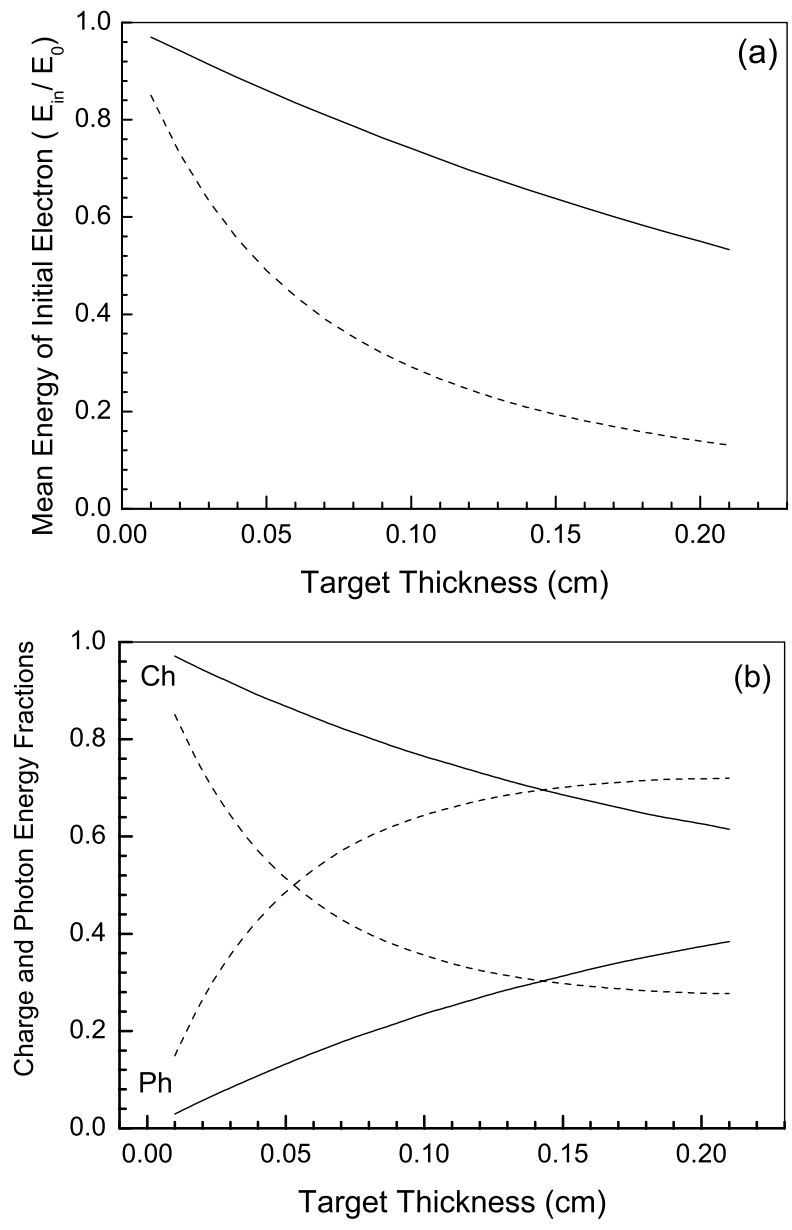

FIG. 1. Mean energy of the initial electron in units of the incident beam energy, $E_{0}=10 \mathrm{GeV}$ (a), and fractions of the total energy carried correspondingly by all charged particles and photons (b). Solid lines are for amorphous and dashed lines for crystal tungsten target. The incident beam is directed along the $\langle 111\rangle$ axis of the crystal.

targets is always less in the crystal case. Let us present some qualitative arguments to explain this fact. The main process leading to the energy deposition is the ionization loss, the rate of which, $q(z)$, reads approximately as $q(z) \simeq$ $C_{Q} N_{\mathrm{ch}}(z)$, where $N_{\mathrm{ch}}(z)$ is the number of charged particles at the depth $z$. Strictly speaking, the coefficient $C_{Q}$ may depend on $z$ but its small variation as well as a small difference of $C_{Q}$ values in crystal and amorphous cases is neglected in our estimation. So, the total energy, $Q(L)$, deposited over the thickness $L$ reads

$$
Q(L)=\int_{0}^{L} d z q(z) \simeq C_{Q} \int_{0}^{L} d z N_{\mathrm{ch}}(z),
$$

or, going over to the variable $N_{\mathrm{ch}}(z)$

$$
Q(L) \simeq C_{Q} \int_{1}^{N_{\mathrm{ch}}(L)} d N_{\mathrm{ch}}\left(\frac{d \ln N_{\mathrm{ch}}}{d z}\right)^{-1} .
$$

For sufficiently large $L$, the positron yield is roughly 
proportional to the total number of charged particles, $N_{\text {ch }}(L)$, i.e., for equivalent targets, the integrals in (2) are taken over the same region of variable $N_{\mathrm{ch}}$ in both cases. To prove our statement, it remains only to verify that the logarithmic derivative which appears in the denominator of the integrand in (2) is larger for crystals. This derivative, or logarithmic increment, characterizes the growth of the number of charged particles. As seen in Fig. 1, the conversion of the initial electron energy into photons is going faster for crystals, where, correspondingly, the pair-production process starts earlier and is more intensive, resulting in a larger increment. For the purposes of illustration, the energy-deposition rate per charged particle and the logarithmic increment are shown in Fig. 2. We emphasize that the energy-deposition rate is practically the same for crystal and amorphous samples, being almost independent of the electron energy. The nonconstancy of this rate is mainly due to the contributions of other processes like photon absorption and annihilation of positrons which are taken into account by our simulations but were ignored in the estimation of $q(z)$
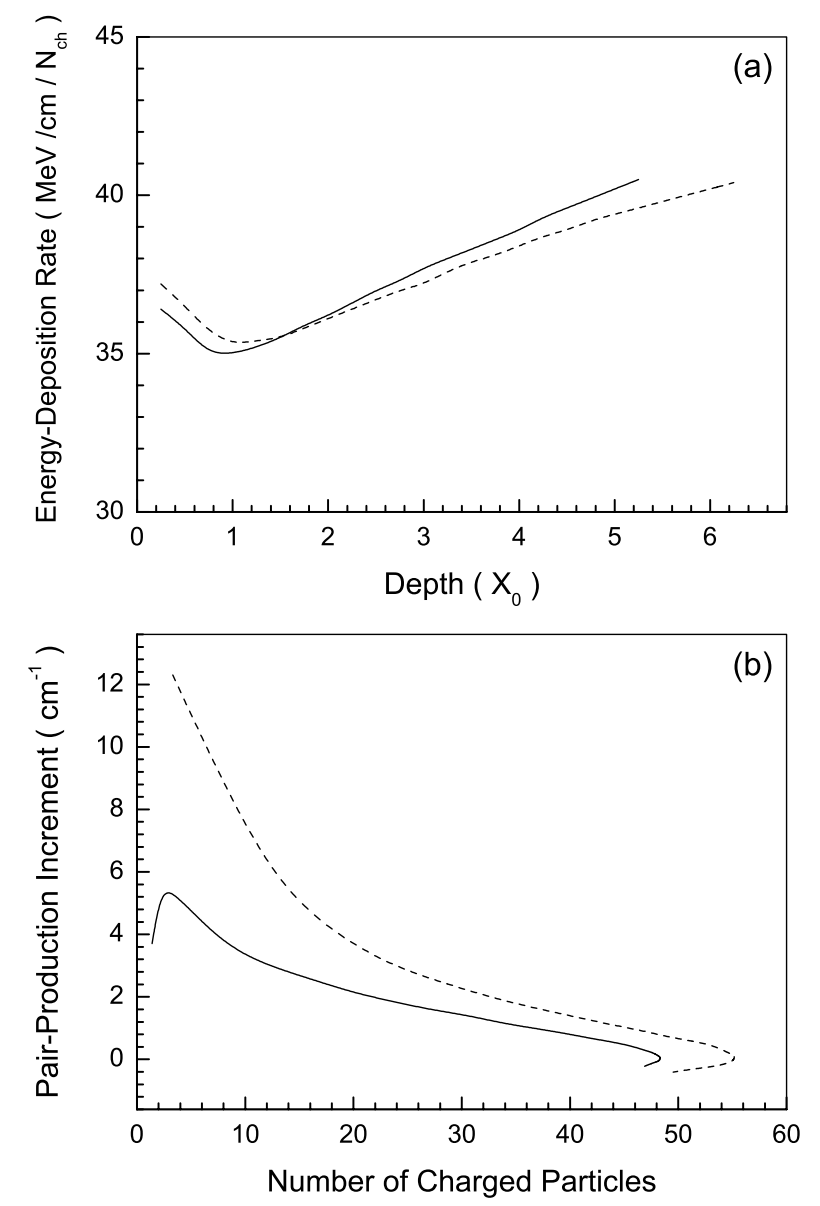

FIG. 2. Energy deposition rate per charged particle (a) and the logarithmic increment (b) in tungsten targets at $E_{0}=$ $10 \mathrm{GeV}$. Solid lines are for amorphous and dashed lines for crystal targets. used in (1). Evidently, the role of these processes increases with growing depth. A slow decrease of the rate at comparatively small depth is due to that of the mean particle energy. The point is that we use the so-called nonrestricted energy loss description, where the rate diminishes when the particle energy does so. On the whole, the results of simulations presented in Fig. 2 confirm the above qualitative considerations.

As a shower develops, particles of sufficiently low energy may substantially change their direction of propagation due to single or multiple scattering, so that there even appears a "backward" flux of particles moving upstream. They do not increase the positron yield; however, they heat the target. This contribution is taken into account in our simulations, where we are able to trace separately effects from "forward" and backward particles and photons. To study the shower characteristics depending on depth $(z)$, the target is divided into slices by planes perpendicular to the incident beam direction, taken as $z$ axis. The spacing $d z=0.25 X_{0}$ is used (remember that $X_{0}=3.5 \mathrm{~mm}$ for tungsten). Energy deposition is simulated within each slice and various distributions are recorded at the right-hand boundary (plane) of the slice. In particular, the development of momentum distributions for positrons and photons is obtained as well as that of the beam spot size. Shown in Fig. 3(a) is the energy, $\Delta E_{\text {dep }}(z)$, deposited in slices in units of $E_{0}$. This quantity is higher for the lower energy. Note that the deposited power, $P_{\text {dep }}$, reads as $P_{\text {dep }}=P_{0} E_{\text {dep }} / E_{0}$ where $P_{0}$ is the incident beam power. The contribution of the backward particles to $E_{\mathrm{dep}}$ increases with the target thickness $L$; it amounts typically to about $20 \%$ at $L \sim 4 X_{0}$ and thereby cannot be neglected. The results of simulations presented in Fig. 3(b) clearly confirm once more (cf. Fig. 9 in [7]) the statement concerning the comparison between the values of $E_{\mathrm{dep}}$ in the equivalent targets. The positron yield is calculated using the "theoretical" acceptance conditions from [2] [see Figs. 11 and 12(b) in [2]] in all three cases. The angular spread of the incident beam is neglected and the transverse size of the beam $\sigma\left(\sigma_{x}=\right.$ $\sigma_{y}=\sigma$ ) is set to $1.6 \mathrm{~mm}$ at 2 and $6.2 \mathrm{GeV}$, and to $2.5 \mathrm{~mm}$ at $10 \mathrm{GeV}$. At $E_{0}=2 \mathrm{GeV}$, a simulation for $\sigma=2.0 \mathrm{~mm}$ was performed as well. Corresponding results, not shown on graphs, are presented in the Table below.

Let us remind one now that, at equal depths and initial energies, charged particles are softer and have a larger angular spread for crystal targets (see, e.g., Figs. 2-5 in [18] and the corresponding discussion). All other things being equal, positron spectra are softer at a lower initial energy. As an illustration of these features, the mean energy and the transverse momentum, $\left\langle p_{t}\right\rangle$, of forward positrons are shown in Fig. 4(a). We emphasize that, starting with $L \sim X_{0},\left\langle p_{t}\right\rangle$ is almost constant and practically independent of the initial electron energy and the type of the target [note the merging of six different curves in Fig. 4(a)]. 

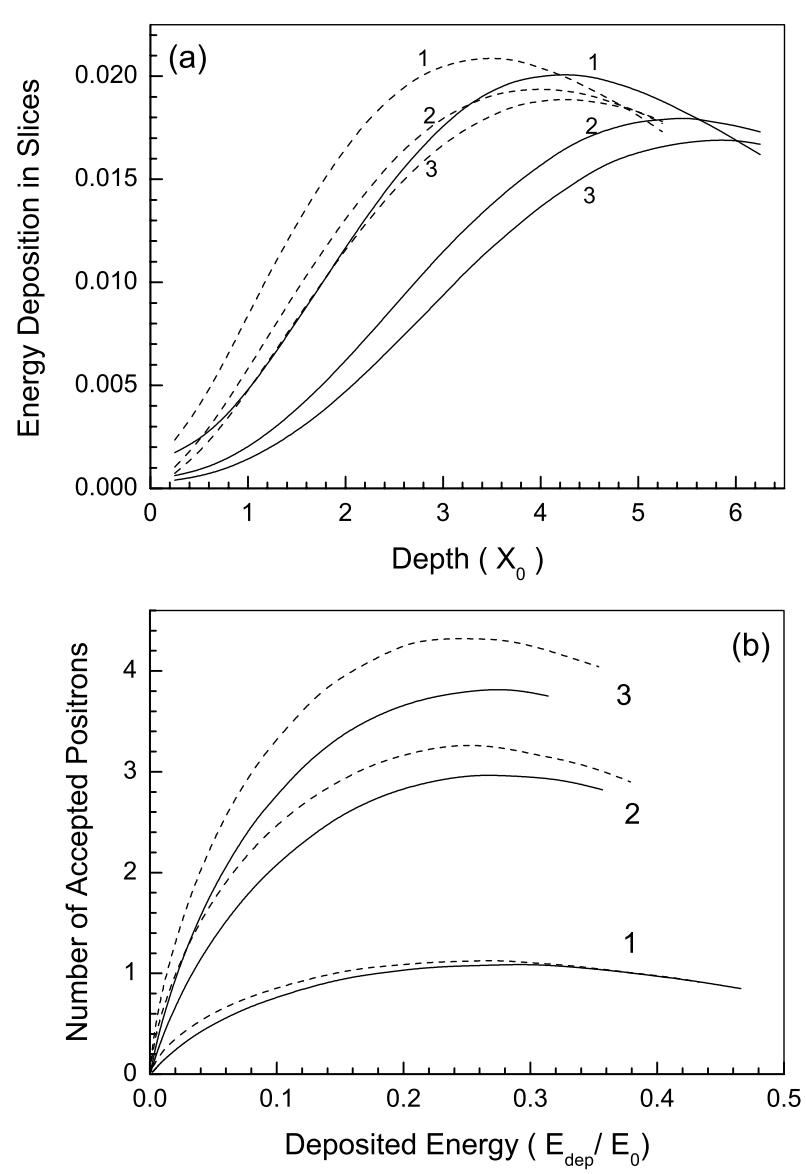

FIG. 3. Fraction of energy $\Delta E_{\text {dep }}(z) / E_{0}$ deposited in $z$ slices (a) and number of accepted positrons as a function of the deposited energy $E_{\mathrm{dep}} / E_{0}$ (b) at $E_{0}=2 \mathrm{GeV}$ (curves 1), 6.2 GeV (curves 2), and $10 \mathrm{GeV}$ (curves 3). Solid lines are for amorphous and dashed lines for crystal targets.

Such results confirm and extend those concerning an amorphous target; it is essentially due to a counterbalance between the increase of the angle as a consequence of the multiple scattering and the decrease of the positron energy with increased thickness [9]. At the same time, the larger angular spread leads in the crystal case to a larger beam spot area, $S$, as seen in Fig. 4(b), where $S / S_{0}$ is plotted for forward charged particles. For evident reasons, the spot area of the backward charged flux (not shown in Fig. 4) is somewhat larger (typically by $20 \%-$ $25 \%$ ) than that of the forward one.

Whereas the average deposited power can be handled somehow (e.g., by rotating the target and removing the excess heat through water cooling as used at SLC), the local and nearly instantaneous energy deposition is unavoidable, while being of critical concern for target damage. Basing on the analysis of the SLC damaged target (see [1]), it is now adopted that the peak energydeposition density should not exceed $35 \mathrm{~J} / \mathrm{g}$ to ensure a sufficiently long term of safe operation. In our simulations, the total scanned volume is a cylinder coaxial with
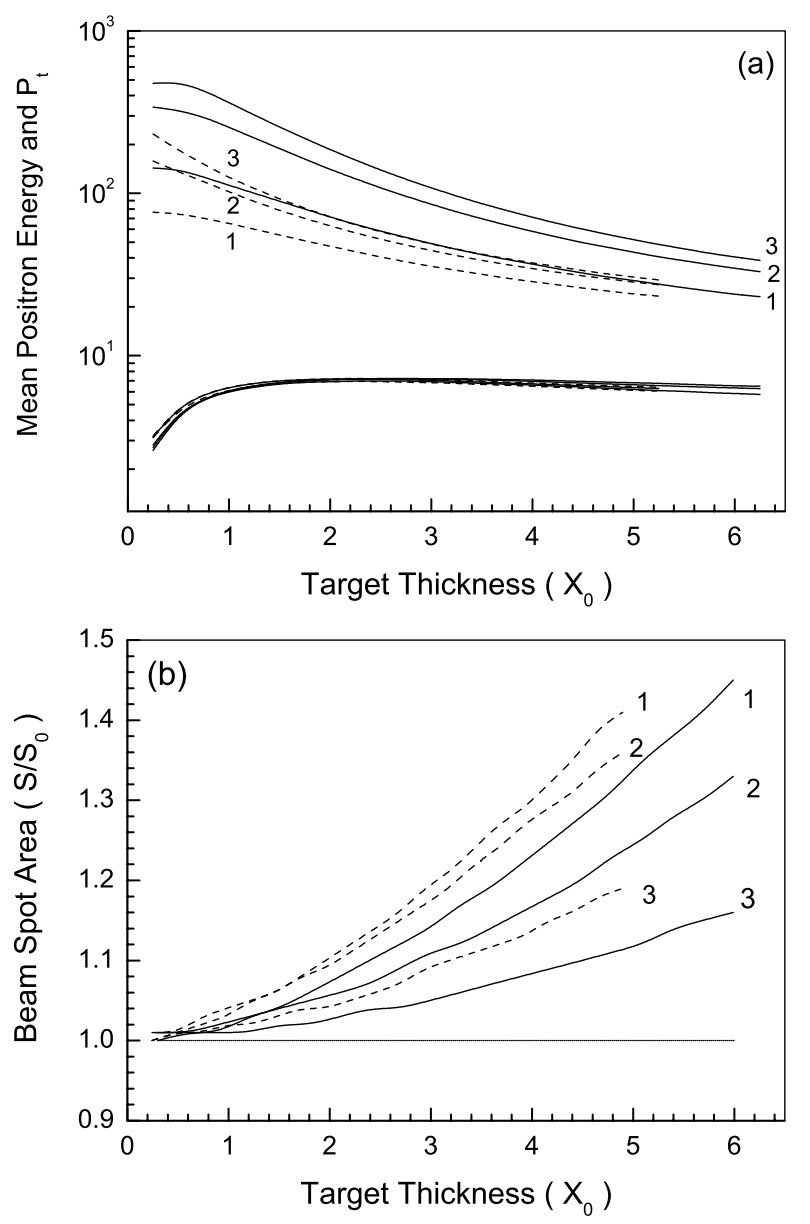

FIG. 4. (a) Mean positron energy (in MeV, upper curves) and transverse momentum (in $\mathrm{MeV} / c$, lower curves); (b) beam spot area development. Incoming energies are $E_{0}=2 \mathrm{GeV}$ (curves 1), $6.2 \mathrm{GeV}$ (curves 2), and $10 \mathrm{GeV}$ (curves 3). $S_{0}$ corresponds to the incident beam. The straight line $y=1$ on (b) is drawn to guide the eye. Solid lines are for amorphous and dashed lines for crystal targets.

the incident beam direction. The radius, $R$, of the cylinder was about $R \simeq 3.7 \sigma$, in which case less than one-thousandth of initial electrons does not hit the cylinder at entry. This cylinder is sliced into disks of the thickness $d z=0.25 X_{0}$, i.e., $z$ spacing is the same as in the above calculations, allowing a mutual checking. In turn, each disk is divided by circles of uniformly increasing radii with the step $d r=0.02 R$ into 50 parts - one internal disk (altogether, such disks form the internal cylinder ) and 49 rings. More precisely, we have $d r(\sigma=1.6 \mathrm{~mm})=$ $0.12 \mathrm{~mm}, \quad d r(\sigma=2.0 \mathrm{~mm})=0.15 \mathrm{~mm}, \quad$ and $d r(\sigma=$ $2.5 \mathrm{~mm})=0.185 \mathrm{~mm}$. The energy deposition and the number of charged particles are simulated in each meshed volume providing corresponding transverse distributions for each $z$ slice. It is noteworthy that $z$ dependencies of shower characteristics derived from 3dimensional distributions coincide with those obtained in direct calculations, thereby verifying the consistency 

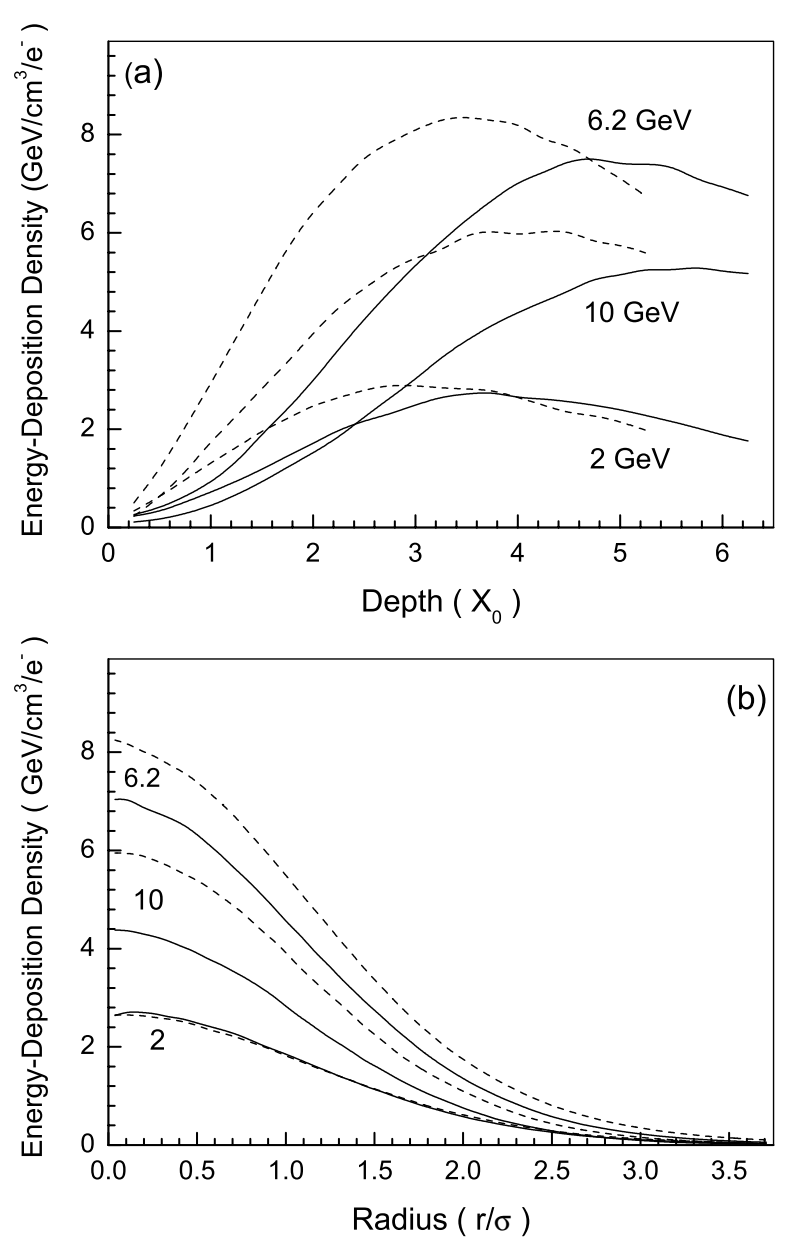

FIG. 5. (a) Energy-deposition density in the internal cylinder, versus the depth, at indicated initial energies; (b) transverse distribution of the energy-deposition density at $z=4.0 X_{0}$. Solid lines are for amorphous and dashed lines for crystal targets.

of the two essentially different forms of the output which we have used.

From Fig. 5(b), the EDD drops at large cell radius, being maximal in the internal disk of each slice. Shown in Fig. 5(a) is the EDD per one incident electron in the internal cylinder, versus the depth. Like the positron yield and the total number of charged particles, the peak values of EDD (PEDD) in crystals are somewhat higher and are reached at smaller depths, compared with amorphous targets. At equal beam size, PEDD is higher for the higher energy [cf. pairs of curves in Fig. 5(a) calculated at 2 and $6.2 \mathrm{GeV}$ for the same $\sigma=1.6 \mathrm{~mm}$ ]. However, by increasing the beam size, lower values of PEDD may be obtained even at higher energy. This is seen in Fig. 5(a) if we compare the curves calculated at $10 \mathrm{GeV}(\sigma=$ $2.5 \mathrm{~mm}$ ) with those at $6.2 \mathrm{GeV}$. Examples of transverse distributions of EDD are plotted in Fig. 5(b) for the same depth $z=4 X_{0}$. The distribution of charged particle density (not shown) is similar to that of EDD with a ratio EDD/density being almost constant within the first ten cells and roughly equal to the energy-deposition rate. In Fig. 5(b) this similarity reveals in a larger width of distributions for crystal targets [cf. Fig. 4(b)]. The EDD is presented in Fig. 5 in units of $\mathrm{GeV} \mathrm{cm}^{-3}$ which for tungsten corresponds to $1 \mathrm{GeV} \mathrm{cm}^{-3}=8.30 \times$ $10^{-12} \mathrm{~J} / \mathrm{g}$.

Because of an extremely short duration of the pulse, contributions to EDD from all the incident electrons are added and the resulting EDD value is simply the product of the obtained EDD value per $e^{-}$and the number of electrons per pulse, provided that the target reverts to initial thermal conditions during the repetition period. Some results concerning the energy deposition in crystal and amorphous targets are presented in Table I. For the sake of comparison, the thicknesses of amorphous tungsten targets $\left(W_{\mathrm{am}}\right)$ are the same as proposed for $\mathrm{W}_{75} \mathrm{Re}_{25}$ targets in the projects [2-4]. For crystal targets $\left(W_{\text {cr }}\right)$, the corresponding thicknesses are determined from the equivalence rule (the same positron yield as for $W_{\mathrm{am}}$ target). Our values for PEDD given in Table I are obtained using the same numbers of electrons per pulse, $N_{e}(2 \mathrm{GeV})=2.08 \times 10^{12}, \quad N_{e}(6.2 \mathrm{GeV})=0.96 \times 10^{12}$, and $N_{e}(10 \mathrm{GeV})=0.64 \times 10^{12}$, as in [2-4], respectively.

Since the parameters of the two amorphous targets such as radiation lengths and densities almost coincide, the same is expected for shower characteristics at equal depths. However, one should bear in mind that two different computer codes were used in simulations, EGS4 for $\mathrm{W}_{75} \operatorname{Re}_{25}$ targets, and our code for $W_{\mathrm{am}}$ targets. From Table I, the concordance of the two approaches is almost

TABLE I. Energy deposition in crystal and amorphous targets. The fraction of an incident beam power deposited in a target, $R_{\mathrm{th}}=P_{\text {dep }} / P_{0}$; the peak energy deposition density, PEDD, in units of $\mathrm{J} / \mathrm{g}$; the energy, $E_{0}$, and transverse size of an incident beam, $\sigma$; target thickness, $L$, measured in conventional radiation lengths, $X_{0}$; gain in the total deposited power, $G=\left(1-E_{\mathrm{cr}}^{\mathrm{dep}} / E_{\mathrm{am}}^{\mathrm{dep}}\right) \cdot$ 100 , from comparison of $W_{\mathrm{cr}}$ and $W_{\mathrm{am}}$ targets.

\begin{tabular}{|c|c|c|c|c|c|c|c|c|c|}
\hline \multirow{2}{*}{$\begin{array}{l}\text { Beam } \\
\text { Target }\end{array}$} & \multicolumn{3}{|c|}{$E_{0}=2 \mathrm{GeV}, \sigma=2.0 \mathrm{~mm}$} & \multicolumn{3}{|c|}{$E_{0}=6.2 \mathrm{GeV}, \sigma=1.6 \mathrm{~mm}$} & \multicolumn{3}{|c|}{$E_{0}=10 \mathrm{GeV}, \sigma=2.5 \mathrm{~mm}$} \\
\hline & $\mathrm{W}_{75} \mathrm{Re}_{25}$ & $W_{\mathrm{am}}$ & $W_{\mathrm{cr}}$ & $\mathrm{W}_{75} \mathrm{Re}_{25}$ & $W_{\mathrm{am}}$ & $W_{\mathrm{cr}}$ & $\mathrm{W}_{75} \mathrm{Re}_{25}$ & $W_{\mathrm{am}}$ & $W_{\text {cr }}$ \\
\hline$L$ & 4.0 & 4.0 & 3.0 & 4.0 & 4.0 & 2.5 & 6.0 & 6.0 & 3.0 \\
\hline$R_{\mathrm{th}}$ & 0.248 & 0.238 & 0.193 & 0.142 & 0.147 & 0.106 & 0.310 & 0.291 & 0.137 \\
\hline PEDD & 35.0 & 32.3 & 33.8 & 35.0 & 56.1 & 60.3 & 35.0 & 28.2 & 30.6 \\
\hline$G$ & & $19 \%$ & & & $28 \%$ & & & $53 \%$ & \\
\hline
\end{tabular}


perfect for the fraction of the deposited power, $R_{\mathrm{th}}$. Concerning the PEDD, the values obtained for the $W_{\mathrm{am}}$ target at 2 and $10 \mathrm{GeV}$ are somewhat smaller than those for the $\mathrm{W}_{75} \mathrm{Re}_{25}$ target and the distinctions are not too big. On the contrary, our value for the $W_{\text {am }}$ target is substantially larger at $6.2 \mathrm{GeV}$. Let us argue the point in detail. At equal beam size, the peak EDD/electron is expected to be roughly proportional to the initial electron energy, $E_{0}$. This assertion follows from the qualitative consideration performed above and is verified by calculations. For example, if we compare the curves in Fig. 5(a) calculated at 2 and $6.2 \mathrm{GeV}$ for the same $\sigma=1.6 \mathrm{~mm}$, we obtain $K=2.7$ as the peak EDD ratio instead of $K=3.1$ from the rough estimate, where $K\left(E_{02}, E_{01}\right)=E_{02} / E_{01}$. At $L=$ $4 X_{0}$, which corresponds to NLC conditions, this ratio further diminishes up to 2.55 since the peak EDD value is achieved for $E_{0}=6.2 \mathrm{GeV}$ at a larger depth. So, at equal beam size, the relationship between PEDD values at different energies reads roughly as

$$
\operatorname{PEDD}(2) \simeq \operatorname{PEDD}(1) \cdot K\left(E_{02}, E_{01}\right) \cdot N_{e}\left(E_{02}\right) / N_{e}\left(E_{01}\right) .
$$

The PEDD in the $\mathrm{W}_{75} \mathrm{Re}_{25}$ target at $E_{0}=2 \mathrm{GeV}$ was calculated in [2] not only for $\sigma=2.0 \mathrm{~mm}$, but also for $\sigma=1.6 \mathrm{~mm}$, where the value of $53.1 \mathrm{~J} / \mathrm{g}$ has been obtained (at these conditions we have $47.7 \mathrm{~J} / \mathrm{g}$ for the $W_{\mathrm{am}}$ target). Using the estimate (3) with $\operatorname{PEDD}(1)=53.1 \mathrm{~J} / \mathrm{g}$ and $K=2.55$, we obtain for NLC conditions $\operatorname{PEDD}(2)=$ $62.5 \mathrm{~J} / \mathrm{g}$ which is consistent with our result for the $W_{\mathrm{am}}$ target. Conversely, starting with the results of [4] for the $\mathrm{W}_{75} \mathrm{Re}_{25}$ target, we obtain the estimate $[\operatorname{PEDD}(1)=$ $\left.35 \mathrm{~J} / \mathrm{g}, E_{01}=10 \mathrm{GeV}, K=0.62\right] \operatorname{PEDD}(2) \simeq 33 \mathrm{~J} / \mathrm{g}$ at $\sigma=2.5 \mathrm{~mm}, E_{02}=6.2 \mathrm{GeV}$. So, the PEDD value of $35 \mathrm{~J} / \mathrm{g}$ at $E_{0}=6.2 \mathrm{GeV}$ is more consistent with the beam size of $\sigma=2.5 \mathrm{~mm}$ than with $\sigma=1.6 \mathrm{~mm}$ indicated in [3].

Comparing the magnitude of thermal effects in $W_{\mathrm{am}}$ and $W_{\text {cr }}$ targets providing the same positron yield, we conclude that, using crystal targets, the total deposited energy can be considerably diminished while the peak value of the energy-deposition density is kept approximately on the same level as in the amorphous case.

\section{ACKNOWLEDGMENTS}

One of us (V.S.) is thankful for kind hospitality during his stay at LAL where a part of this work was done. He is also grateful to the Russian Fund of Basic Research for partial support of this work by Grants No. 01-02-16926 and No. 03-02-16510.

[1] S. Maloy et al., LANL Report No. LA-UR-01-1913, 2001.

[2] T. Kamitani and L. Rinolfi, CERN, CLIC Note No. 465, 2001.

[3] SLAC Report No. SLAC-R-571, 2001, edited by N. Phinney.

[4] T. Kamitani, in Proceedings of the Mini-Workshop on Channeling Radiation Phenomena and Positron Production, KEK Proc. No. 2002-26 (KEK, Tsukuba, 2003), pp. 95-101.

[5] R. Medenwaldt et al., Phys. Lett. B 227, 483 (1989).

[6] V. N. Baier, V. M. Katkov, and V. M. Strakhovenko, Nucl. Instrum. Methods Phys. Res., Sect. B 27, 360 (1987).

[7] V. N. Baier, V. M. Katkov, and V. M. Strakhovenko, Nucl. Instrum. Methods Phys. Res., Sect. B 103, 147 (1995).

[8] V. N. Baier, V. M. Katkov, and V. M. Strakhovenko, Nucl. Instrum. Methods Phys. Res., Sect. B 119, 131 (1996).

[9] X. Artru, V. N. Baier, R. Chehab, and A. Jejcic, Nucl. Instrum. Methods Phys. Res., Sect. A 344, 443 (1994).

[10] V. N. Baier and V. M. Strakhovenko, Nucl. Instrum. Methods Phys. Res., Sect. B 155, 403 (1999.

[11] R. Chehab et al., in Proceedings of the IEEE Particle Accelerator Conference, Chicago, 1989 (IEEE, Piscataway, NJ, 1989), pp. 283-285.

[12] X. Artru et al., Nucl. Instrum. Methods Phys. Res., Sect. B 119, 246 (1996).

[13] K. Yoshida et al., Phys. Rev. Lett., 80, 1437 (1998).

[14] M. Inoue et al., Nucl. Instrum. Methods Phys. Res., Sect. B 173, 104 (2001).

[15] R.Chehab et al., Phys. Lett. B 525, 41 (2002).

[16] X. Artru et al., Nucl. Instrum. Methods Phys. Res., Sect. B 201, 243 (2003).

[17] H. Okuno et al., Nucl. Instrum. Methods Phys. Res., Sect. B 201, 259 (2003).

[18] V. N. Baier and V. M. Strakhovenko, Phys. Rev. ST Accel. Beams, 5, 121001 (2002). 